\title{
Diseño de interfaces de inspección de calidad utilizando redes neuronales y visión artificial para la industria de manufactura
}

\author{
Luis Arturo Medina Muñoz, Samuel González-López, \\ Jesús Antonio Mayorquín Robles, Gabriel Antonio López Valencia \\ Instituto Tecnológico de Nogales, División de Estudios de Posgrado e Investigación, \\ Nogales, Sonora, México \\ mmarturo756@gmail.com, samuelgonzalezlopez@gmail.com, \\ ing.mayo@hotmail.com, glopez09@hotmail.com
}

\begin{abstract}
Resumen. La inspección de componentes con sistemas de visión es una tarea que se puede lograr con diferentes métodos. La inspección en la mayoría de los procesos de fabricación depende principalmente de operadores humanos cuyo rendimiento es generalmente inadecuado y variable [4]. Las áreas de manufactura donde la visión juega un papel importante son la inspección, las mediciones y algunas tareas de ensamblaje [5]. Este artículo aborda el problema implementando redes neuronales artificiales. Utilizamos la técnica de erosión de imagen contra una imagen maestra (conector) y el reconocimiento de patrones de redes neuronales después de la erosión. La aceptabilidad de los conectores o cables se logra utilizando el reconocimiento de elementos mediante visión artificial. En este trabajo, se explica cómo podemos extraer las características clave de la imagen, posición de las chavetas del conector, y las convertimos en números y alimentamos la red neuronal para determinar si un conector o cable cumple con los requisitos o es un elemento defectuoso. El sistema se validó en el área de inspección de recibo en una fábrica local de manufactura, donde se realiza la primera inspección de los componentes, en contra de su dibujo y en la línea de producción. Los resultados de la validación del sistema de visión muestran una aceptación del $100 \%$ para la identificación de conectores y una exactitud de 83\% para la identificación de cable.
\end{abstract}

Palabras claves: sistemas de inspección, industria de manufactura, redes neuronales, sistemas de visión.

\section{Design of Quality Inspection Interfaces using Neural Networks and Artificial Vision for the Manufacturing Industry}

\begin{abstract}
The inspection of components with vision systems is a task that can be achieved with different methods. This article addresses the problem by implementing artificial neural networks. We use the technique of image erosion against a master image (connector) and the recognition of neural network patterns after erosion. The acceptability of the connectors or cables is achieved using the recognition of elements by artificial vision. In this paper, we explain how we can
\end{abstract}


extract the key features of the image, position the connector keys, and convert them into numbers and feed the neural network to determine if a connector or cable meets the requirements or is a defective element. The system was validated in the area of receipt inspection in a local manufacturing factory, where the first inspection of the components is carried out, against their drawing and in the production line. The results of the validation of the vision system show a $100 \%$ acceptance for the identification of connectors and an accuracy of $83 \%$ for the identification of the cable.

Keywords: inspection systems, manufacturing industry, neural networks, vision systems.

\section{Introducción}

La inspección industrial es una de las tareas cruciales para garantizar la calidad de los productos [6]. El menor costo de mano de obra en los países en desarrollo en comparación con el de las economías desarrolladas a menudo resulta difícil para justificar la maquinaria de automatización. Si bien esta tecnología puede mejorar la calidad del producto y la productividad, así como reducir el costo de los productos, en las economías en desarrollo y emergentes, a menudo es necesario que las empresas manufactureras soliciten apoyo a centros de investigación, ya que las universidades se dirigen a los estudiantes para apoyar a las industrias locales [1].

La Visión Artificial (AV) utiliza imágenes que son capturadas por una cámara de visión industrial y luego procesadas a través de software específico.

De esta manera, $\mathrm{AV}$ puede medir, contar, verificar, seleccionar e identificar fallas y anomalías y, con base en este procesamiento, la tecnología también tiene la capacidad de tomar decisiones como expulsar un determinado producto de la línea de producción cuando éste no cumpla con los estándares de calidad exigidos. Estas decisiones se toman en base a parámetros preestablecidos, de modo que todo lo que no cumpla con estos parámetros se considera incorrecto [2].

El precio de un sistema de visión en el mercado tiende a oscilar entre 2,000 y 100,000 USD. La industria de fabricación local tiene áreas de inspección de recibos y de producción, por lo que el costo para un sistema de visión podría ser tan alto como 60,000 USD, además de otros 15,000 USD para trabajos de calibración y entrenamiento de personal por año debido a la naturaleza cambiante del entorno de trabajo.

El objetivo de este artículo es proponer el desarrollo de un sistema de inspección automática de bajo costo utilizando técnicas de visión y redes neuronales artificiales entrenadas con el algoritmo de propagación hacia atrás, capaz de reducir los errores humanos. El sistema de inspección deberá ser rápido y confiable, capaz de identificar conectores con las características deseadas correctas y el despoje adecuado de los cables, además de otras características importantes en conectores y cables.

\section{Marco referencial}

Las redes neuronales artificiales (RNN) se han desarrollado como una generalización de los modelos matemáticos de la cognición humana y se han mostrado 
prometedores para resolver problemas difíciles en áreas como el reconocimiento de patrones y la clasificación. Una de las principales dificultades que enfrentan los investigadores que usan redes neuronales es la selección del tamaño y la topología adecuados de las redes [7].

La obtención de buenos resultados depende en gran medida de los parámetros de aprendizaje, de los pesos iniciales, así como la topología de la red [10]. El uso de redes neuronales como clasificador requiere una fase de entrenamiento y una fase de prueba. En la fase de entrenamiento, la red neuronal realiza el ajuste apropiado para sus pesos (W) para producir la respuesta deseada. Cuando la respuesta de salida real se aproxima a la deseada, la red ha completado el entrenamiento. En la fase de prueba, se solicita a la red neuronal que clasifique un nuevo conjunto de imágenes y se evalúa su éxito [3].

En [9] los autores abordaron la tarea de identificar defectos o imperfecciones de la superficie en los procesos de fundición, como la inclusión, la vuelta fría y los errores. Los autores utilizaron un método de segmentación que marca la región afectada por algunos de los defectos y luego aplicaron técnicas de aprendizaje automático para clasificar las regiones incorrectas.

Los investigadores utilizaron la herramienta Weka (Weka es una plataforma de software para el aprendizaje automático y minería de datos) para realizar la tarea de clasificación con los algoritmos NaiveBayes, SVM, J48 KNN y RandomForest (RF). Con RF y una muestra de 50 los autores lograron una precisión de aproximadamente el 95\%. De manera similar, nuestro trabajo busca identificar defectos en materiales diversos para luego predecir con el uso de una red neuronal.

El mantenimiento de los estándares de calidad es importante en cualquier empresa debido a la rotación de los empleados en el área de inspección y producción. En [10], los autores proponen un modelo de red neuronal para ayudar a los profesionales de la industria a tomar decisiones en el área de inspección con entradas binarias. La experimentación se llevó a cabo utilizando el $70 \%$ de los datos para el entrenamiento y el $30 \%$ para las pruebas. Los autores realizaron una evaluación en dos etapas. En la primera, a una red neuronal se le permitió decidir si el producto era un rechazo. En la segunda etapa, un segundo modelo de red neuronal decidió si la pieza podía ser reelaborada o si era definitivamente desperdicio.

A diferencia de nuestro trabajo, los autores utilizaron los comentarios de expertos, mientras que este trabajo toma información de las piezas a través de una cámara y hace un análisis similar al que hicieron en [8] donde se muestran datos importantes como la precisión de las redes neuronales, su estructura en términos de la cantidad de entradas, nodos ocultos y salidas. En la fase 1, alcanzaron el $97 \%$ de las piezas correctamente clasificadas y en la fase 2, la precisión fue del $98 \%$.

\section{Desarrollo}

Debido al estricto requisito de calidad y la falta de inspección por visión artificial, es necesario inspeccionar el $100 \%$ de los productos. La empresa utiliza doble inspección en conectores. Durante el proceso de fabricación y la inspección final por control de calidad, pero aún con la doble inspección, la compañía rechazó 16,000 conectores y 300 arneses defectuosos que fueron aceptados por la fabricación y control de calidad en enero y marzo de 2017. El defecto en los conectores, se refiere a un 
corrimiento de entre 1 o 2 grados de la posición original de las chavetas indicadas con el color blanco en la figura 7 como resultado de la binarización de la imagen. El costo de la mala calidad se ha estimado en 50,000 USD. Además, se revisa el despoje de cables blindados utilizados en antenas, que se maquilan en máquinas despojadoras especiales para ello, aun así, existen problemas de calidad.

El sistema propuesto utiliza la cámara de inspección Mighty Scope. La cámara tiene integrado 6 LEDs de alta intensidad; los LED ayudan en el 85\% de los casos para evitar el bajo factor de iluminación. Por otra parte, la cámara fue diseñada para la tarea de inspección. Por esta razón, la lente está dedicada a capturar detalles en la imagen, esto es útil para obtener imágenes para procesar. Para obtener la imagen del conector, la cámara debe permanecer estable, fue necesario utilizar el soporte para cámara "Migthy Scope View Stand”. La figura 1 muestra la cámara y el soporte juntos.

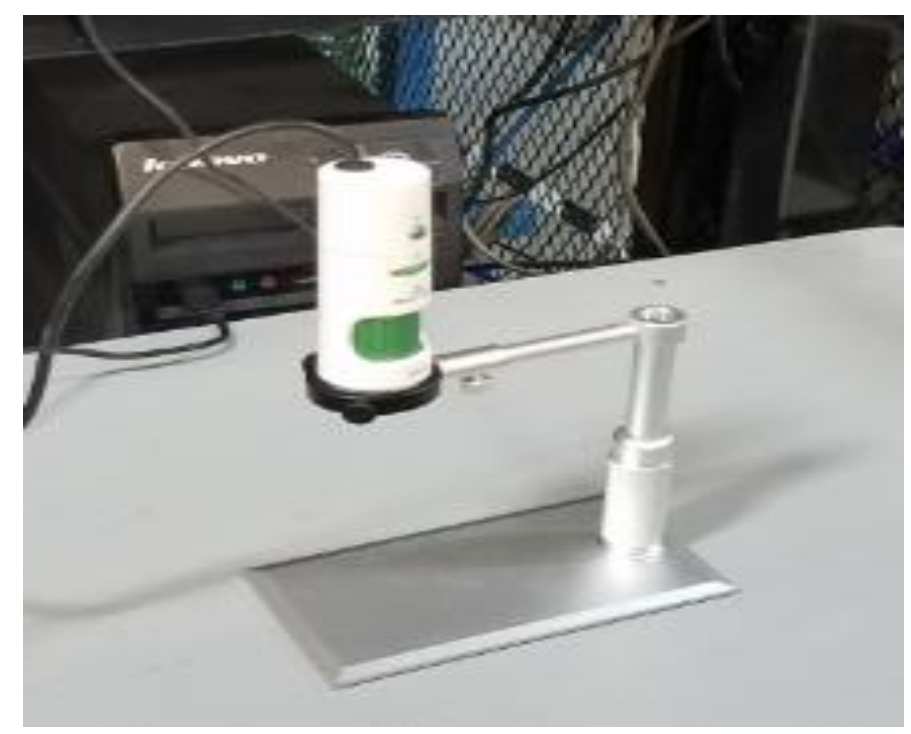

Fig. 1. Cámara de inspección Mighty Scope.

\subsection{Procesamiento de conectores}

La figura 2 muestra el diagrama de flujo del sistema para inspección de conectores.

En el proceso de captura de imagen, aun y cuando existen varios tipos de estos dispositivos, se utilizó una cámara capaz de tomar video y fotografías digitales con iluminación integrada para minimizar los efectos de una iluminación pobre (Fig. 3).

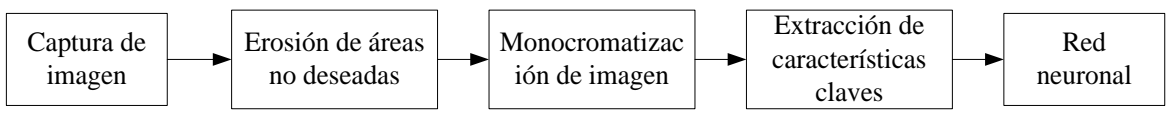

Fig. 2. Diagrama de flujo del Sistema. 
Diseño de interfaces de inspección de calidad utilizando redes neuronales y visión artificial...

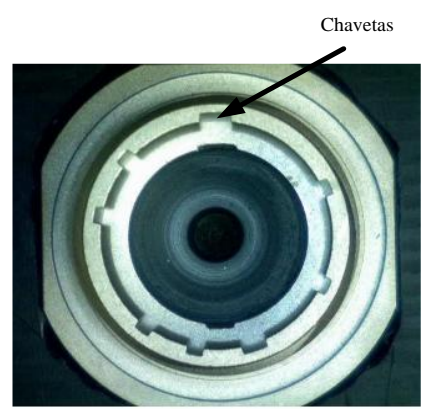

Fig. 3. Imagen de conector a inspeccionar con 8 chavetas.

El proceso de erosión se logra mediante 3 etapas distintas:

1. Dibujo de un círculo interior dentro de las chavetas del conector.

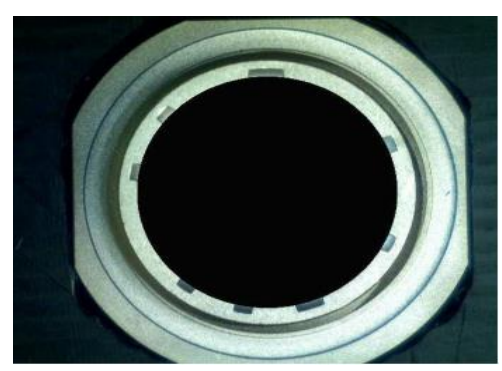

Fig. 4. Dibujo del círculo interior.

2. La figura 5 muestra el dibujo del círculo exterior que cubre el área de interés y deja el área exterior del conector.

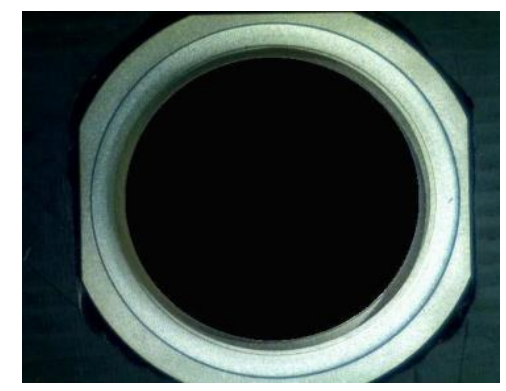

Fig. 5. Dibujo del círculo exterior.

3. La erosión de imágenes se logra comparando las figuras 3 y 4, los pixeles iguales se muestran en color negro dejando solamente los pixeles que son diferentes, como lo muestra la figura 6. 


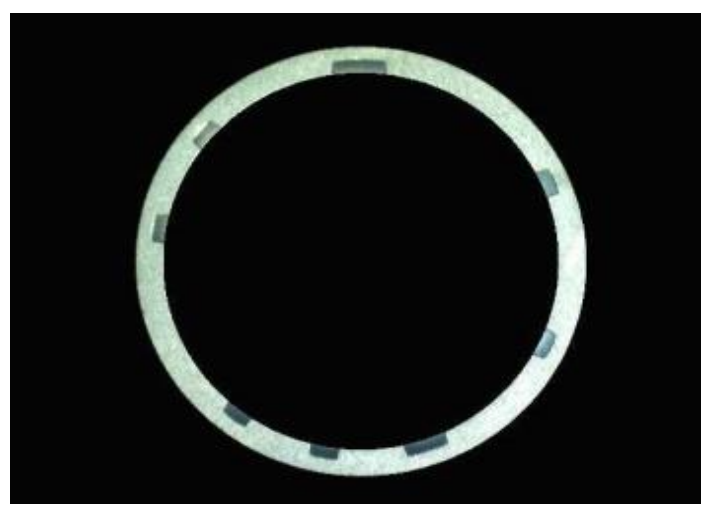

Fig. 6. Imagen erosionada.

El proceso de monocromatización y obtención de características claves se logra haciendo un recorrido de 360 grados desde el centro del conector hasta la altura del radio conocido y los pixeles encontrados como blancos corresponden a la ubicación de la chaveta a inspeccionar. Los datos de salida de extracción como características claves, son expresados como 1, donde se detecta que se encuentra la chaveta, que corresponde al color blanco y un 0 donde no existe la chaveta y que corresponde a un color negro. Esto se muestra en la figura 7 y tabla 1.

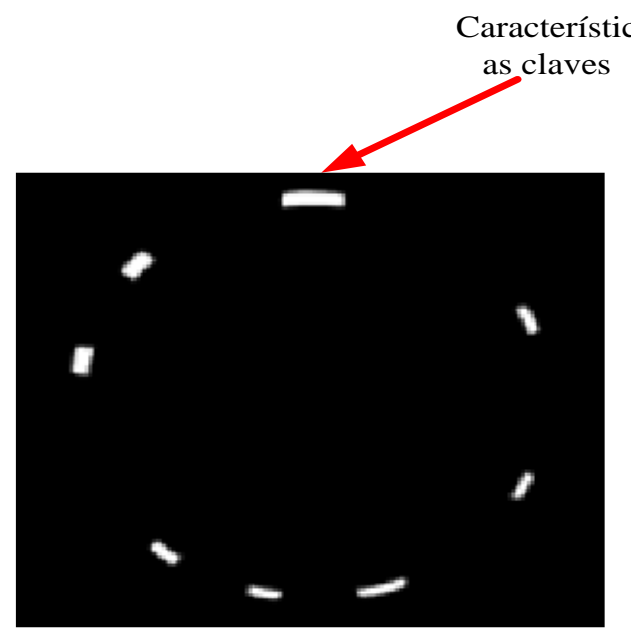

Fig. 7. Imagen procesada.

Los datos de la tabla 1 serán utilizados como parte del entrenamiento de la RNA, la imagen muestra un arreglo de 30 renglones por 12 columnas, de izquierda a derecha son el recorrido de 12 grados del círculo . de arriba hacia debajo de 12 en 12 hasta completar el recorrido de los 360 grados. El 1 significa que se encontró un color blanco que corresponde a la posición de una chaveta y el 0 corresponde a un color negro que indica la ausencia de la chaveta. 
Tabla 1. Imagen binarizada.

$\begin{array}{llllllllllll}0 & 0 & 0 & 0 & 0 & 0 & 0 & 0 & 0 & 0 & 0 & 0 \\ 0 & 0 & 0 & 0 & 0 & 0 & 1 & 1 & 1 & 1 & 1 & 1 \\ 1 & 1 & 0 & 0 & 0 & 0 & 0 & 0 & 0 & 0 & 0 & 0 \\ 0 & 0 & 0 & 0 & 0 & 0 & 0 & 0 & 0 & 0 & 0 & 0 \\ 0 & 0 & 0 & 0 & 0 & 0 & 0 & 0 & 0 & 0 & 0 & 0 \\ 0 & 0 & 0 & 0 & 0 & 0 & 0 & 0 & 0 & 0 & 0 & 0 \\ 0 & 0 & 0 & 0 & 0 & 0 & 0 & 0 & 1 & 1 & 1 & 1 \\ 1 & 1 & 1 & 1 & 1 & 1 & 1 & 1 & 1 & 1 & 1 & 0 \\ 0 & 0 & 0 & 0 & 0 & 0 & 0 & 0 & 0 & 0 & 0 & 0 \\ 0 & 0 & 0 & 0 & 0 & 0 & 0 & 0 & 0 & 0 & 0 & 0 \\ 0 & 0 & 0 & 0 & 0 & 0 & 0 & 0 & 0 & 0 & 0 & 0 \\ 1 & 1 & 1 & 1 & 1 & 1 & 1 & 1 & 1 & 0 & 0 & 0 \\ 0 & 0 & 0 & 0 & 0 & 0 & 0 & 0 & 0 & 0 & 0 & 0 \\ 0 & 0 & 0 & 0 & 0 & 0 & 0 & 0 & 1 & 1 & 1 & 1 \\ 1 & 1 & 1 & 1 & 0 & 0 & 0 & 0 & 0 & 0 & 0 & 0 \\ 0 & 0 & 0 & 0 & 0 & 0 & 0 & 0 & 0 & 0 & 0 & 0 \\ 0 & 0 & 0 & 0 & 0 & 0 & 0 & 0 & 0 & 0 & 0 & 0 \\ 0 & 0 & 0 & 0 & 0 & 0 & 0 & 0 & 0 & 0 & 0 & 0 \\ 0 & 0 & 0 & 0 & 0 & 0 & 0 & 0 & 0 & 0 & 0 & 1 \\ 1 & 1 & 1 & 1 & 1 & 1 & 1 & 0 & 0 & 0 & 0 & 0 \\ 0 & 0 & 0 & 0 & 0 & 0 & 0 & 0 & 0 & 0 & 0 & 0 \\ 0 & 0 & 1 & 1 & 1 & 1 & 1 & 1 & 1 & 1 & 0 & 0 \\ 0 & 0 & 0 & 0 & 0 & 0 & 0 & 0 & 0 & 0 & 0 & 0 \\ 0 & 0 & 0 & 0 & 0 & 0 & 1 & 1 & 1 & 1 & 1 & 1 \\ 1 & 1 & 1 & 1 & 1 & 1 & 0 & 0 & 0 & 0 & 0 & 0 \\ 0 & 0 & 0 & 0 & 0 & 0 & 0 & 0 & 0 & 0 & 0 & 0 \\ 0 & 0 & 0 & 0 & 0 & 0 & 0 & 0 & 0 & 0 & 0 & 0 \\ 0 & 0 & 0 & 0 & 0 & 1 & 1 & 1 & 1 & 1 & 1 & 1 \\ 1 & 0 & 0 & 0 & 0 & 0 & 0 & 0 & 0 & 0 & 0 & 0 \\ 0 & 0 & 0 & 0 & 0 & 0 & 0 & 0 & 0 & 0 & 0 & 0\end{array}$

\subsection{Procesamiento de cables}

La figura 8, nos muestra el diagrama de flujo para la inspección del despoje en cables blindados, indicando el procedimiento a realizar en cada una de ellas en el proceso de la inspección de calidad.

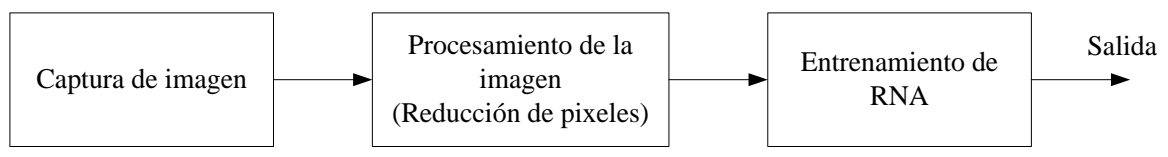

Fig. 8. Diagrama de flujo para la inspección de cables blindados.

Las imágenes fueron procesadas para reducir el tamaño, esto es, la imagen tenía el tamaño de 2,907 x 5,168 Pixeles, la figura 9 muestra la captura original del cable, así que se desarrolló una aplicación para capturar la imagen del cable y reducirla a un tamaño de 600 x 5,168 Pixeles.

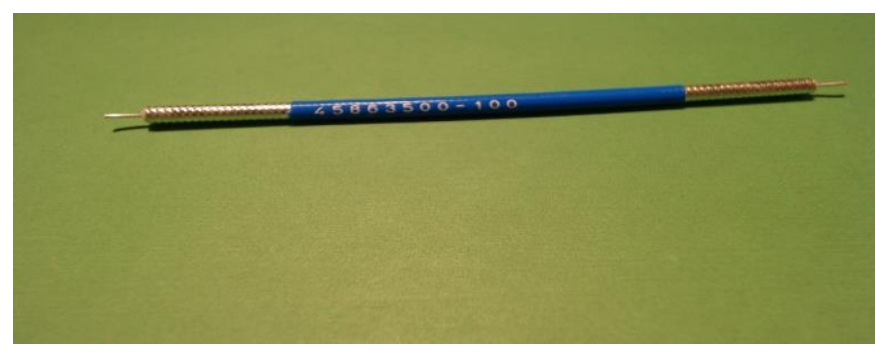

Fig. 9. Imagen del cable a procesar.

De la imagen original se eliminó el fondo verde a través de un filtro, esto redujo el tamaño de la imagen de $1.6 \mathrm{M}$ a $178 \mathrm{~K}$, figura 10, a esta imagen se le aplicó la escala de grises y se sacó el histograma, dando como resultado un archivo con 256 columnas a 
escala de grises, al cual se le agregó otra columna, a las piezas aceptables se le dio el valor de "1" y para las piezas defectuosas el valor de " 0 ".

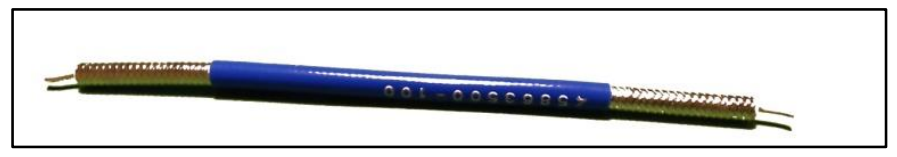

Fig. 10. Imagen procesada.

El entrenamiento de la red neuronal de hizo con los datos del histograma de 190 cables aceptables y con 54 histogramas de cables defectuosos, dejando 10 cables aceptables fuera del entrenamiento, así como 10 cables defectuosos para utilizarse como vector de pruebas.

Se propone implementar una estructura de RNA, como la mostrada en la figura 11, entrenada con el algoritmo de propagación hacia atrás, para realizar el reconocimiento de los patrones a identificar en ambas pruebas, posición de las chavetas en conectores y despoje de cables.

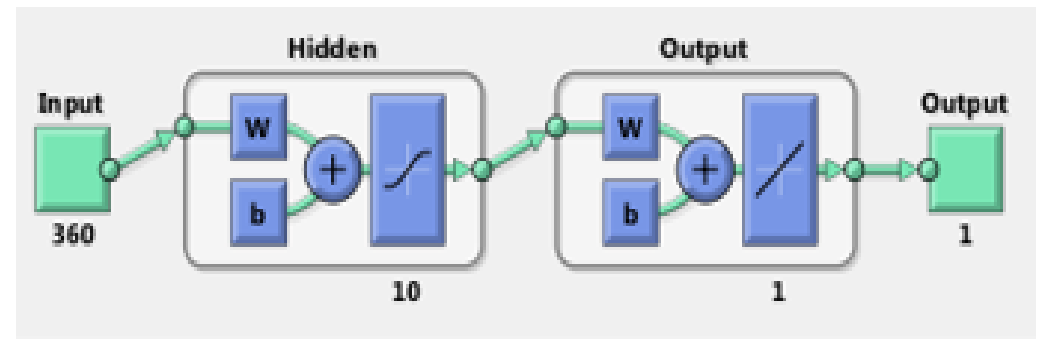

Fig. 11. Estructura de RNA propuesta.

\subsection{Experimentación}

Se utilizó una RNA perceptrón multicapa con un algoritmo de propagación hacia atrás para el entrenamiento, con una cantidad fija de neuronas en la capa oculta para la inspección de los conectores y una estructura de RNA con variación en la cantidad de neuronas en la capa oculta para la inspección de cables. La decisión sobre el uso de este tipo de estructura neuronal se basa en un análisis de prueba de diferentes topologías con un conjunto de datos de entrenamiento y pruebas que satisfacen la política de calidad de la compañía. La Tabla 2 muestra el número de conectores para el entrenamiento y el vector de prueba.

\subsection{Resultados de la inspección de conectores}

Tabla 2. Conjunto de entrenamiento de la RNA.

\begin{tabular}{cc}
\hline Descripción & Cantidad \\
\hline Conectores aceptables & 230 \\
\hline Conectores no aceptables & 50 \\
\hline
\end{tabular}


Finalmente, se usó un lote completo del producto como vector de prueba. En este caso, se utilizaron los conectores bajo prueba, que correspondían a la cantidad de 2,412 conectores en el área de entrada. Cabe mencionar que el lote completo era de conectores válidos y no se tenía control sobre el lote para incluir señuelos. La tabla 3 muestra los resultados de la inspección de prueba.

Tabla 3. Resultados de la inspección.

\begin{tabular}{cc}
\hline Lote de conectores & Resultados de la prueba \\
\hline 2,412 & 2,412 reconocimientos exitosos \\
\cline { 2 - 2 } & 0 rechazos \\
\hline
\end{tabular}

El tiempo de inspección requerido se redujo drásticamente, mostrando una gran mejora cuando se implementa este proceso automático propuesto, como se muestra en la Tabla 4.

Tabla 4. Comparativo de inspección.

\begin{tabular}{cc}
\hline Tipo de inspección & Tiempo de inspección \\
\hline Manual & 150 segundos \\
\hline Automatico & 8 segundos
\end{tabular}

Después de entrenar la RNA propuesta con los datos mostrados en la tabla 2 , podemos ver que el modelo propuesto funciona bien, clasificando sin errores de inspección el número de conectores que se muestra en la Tabla 3, con una tasa de éxito del $100 \%$. Los resultados que se muestran en la Tabla 4 muestran un proceso rápido que reduce el tiempo de inspección en un $95 \%$.

Uno de los objetivos propuestos se ha logrado porque tenemos un sistema de inspección rápido y confiable. Este modelo también muestra que es posible reducir los costos de implementación del sistema en un 94\%, comparando lo que cuesta un sistema industrial y el desarrollo de la aplicación.

Este dispositivo de inspección propuesto, es posible que, se pueda utilizar para verificar la calidad de otros productos utilizando solo los datos de entrenamiento apropiados para la RNA y los resultados se pueden comparar entre sí.

\subsection{Resultados de la inspección de cables}

Para la inspección de cables blindados se realizaron pruebas con la misma estructura de RNA propuesta en la figura 11 del tipo perceptrón multicapa con un algoritmo de propagación hacia atrás para el entrenamiento, variando la cantidad de neuronas en la capa oculta. Se utilizaron los datos mostrados en la tabla 5 para el entrenamiento de la RNA.

Tabla 5. Conjunto de entrenamiento de la RNA.

\begin{tabular}{cc}
\hline Descripción & Cantidad \\
\hline Cables aceptables & 190 \\
\hline Cables con defectos & 54 \\
\hline
\end{tabular}


Luis Arturo Medina Muñoz, Samuel González-López, Jesús Antonio Mayorquín Robles, et al.

Tabla 6. Matriz de confusion de la inspección con 4 neuronas en capa oculta.

\begin{tabular}{ccc}
\hline Aceptables & Defectusos & \\
\hline 179 & 11 & Aceptables \\
\hline 19 & 35 & Defectuosos \\
\hline
\end{tabular}

La medida de exactitud BAC(Balanced Accuracy) obtenida para la configuracion con neuronas en la capa oculta fue de $83.24 \%$.

Tabla 7. Resultados de la inspección con 9 neurona en capa oculta.

\begin{tabular}{ccl}
\hline Aceptables & Defectusos & \\
\hline 163 & 27 & Aceptables \\
\hline 9 & 45 & Defectusos \\
\hline
\end{tabular}

La medida de exactitud BAC(Balanced Accuracy) obtenida para la configuracion con neuronas en la capa oculta fue de $78.63 \%$.

Tabla 8. Matriz de confusion de la inspección con 10 neurona en capa oculta.

\begin{tabular}{ccc}
\hline Aceptables & Defectusos & \\
\hline 137 & 53 & Aceptables \\
\hline 1 & 53 & Defectusos \\
\hline
\end{tabular}

La medida de exactitud BAC(Balanced Accuracy) obtenida para la configuracion con neuronas en la capa oculta fue de $74.63 \%$.

Después de entrenar la RNA variando la cantidad de neuronas en la capa oculta, con la misma cantidad de datos, en el reconocimiento de cables, los resultados tienen variaciones muy representativas para poder definir una estructura apropiada para este tipo de tareas.

En la matriz de confusión de la Tabla 6, podemos observar que la estructura es aceptable para identificar cables con el despoje correcto, pero es pobre en la identificación de cables con defectos. La Tabla 7, muestra que, al modificar el número de neuronas en la capa oculta a 9, la tasa de éxito para cables con despoje correcto es bajo, pero el éxito en la identificación de cables con defecto aumenta, comparado con 4 neuronas. La tabla 7, muestra un equilibrio en el porcentaje éxitos para aceptación y rechazo, pero el porcentaje es bajo para ser tomado como un sistema confiable. La tabla 8 , la cual se obtuvo variando a 10 el número de neuronas en la capa oculta, el porcentaje de éxito es bajo, $74.63 \%$, mostrando que tiene un mejor logro de identificación de cables defectuosos, pero con un pobre desempeño en la clasificación de cables correctos. De acuerdo a estos resultados obtenidos, no es posible definir una estructura de RNA adecuada que cumpla con los criterios de calidad definidos por la empresa de manufactura.

\section{Conclusiones}

Después de entrenar diferentes modelos de RNA con la misma cantidad de datos, podemos ver que el modelo propuesto funciona bien y clasifica sin errores de 
inspección el número de conectores que se muestra en la Tabla 3 , con una tasa de éxito del $100 \%$. Los resultados que se muestran en la Tabla 4 muestran un proceso rápido que reduce el tiempo de inspección en un $95 \%$. Este modelo también muestra que es posible reducir los costos de implementación del sistema en un 94\%, comparando lo que cuesta un sistema industrial y el desarrollo de la aplicación.

La inspección de cables muestra un desempeño pobre en la clasificación de patrones de las estructuras de RNA, es probable que se necesite modificar la cantidad de muestras de entrenamiento o utilizar otros algoritmos para lograr equilibrar el porcentaje de aceptación de cables con las características deseadas y de rechazar los cables que no sean consistentes con esto.

Este dispositivo de inspección propuesto se puede utilizar para verificar la calidad de otros productos utilizando solo los datos de entrenamiento apropiados para la RNA y los resultados se pueden comparar entre sí.

El uso de lenguajes de programación de alto nivel facilita la elaboración de interfaces que coinciden o superan las características de los productos del tipo industrial utilizado para visualizar información. Además, con el desarrollo de la informática, facilitan la incorporación de algoritmos de inteligencia artificial y técnicas de procesamiento de visión que, cuando se utilizan con cámaras de video comerciales, podemos diseñar sistemas de inspección automática. El trabajo futuro implica ampliar el aprendizaje y el reconocimiento con conectores y cables que correspondan a otros modelos e implementar un conjunto de equipos de inspección automatizados en toda la planta de fabricación.

\section{Referencias}

1. Oppenheimer.: El impacto de la automatización en países de ingresos medios. www.semana económica.com. Último acceso: 2018/01/21

2. https://recursos.infaimon.com/hubfs/ebooks/MON_-_eBook. Último acceso: 2018/02/05

3. Banumathi, P., Nasira, G.M.: Fabric Inspection System using Artificial Neural Networks. International Journal of Computer Engineering Science (IJCES), 2(5) (2012)

4. Saravanan, A.: Artificial Neural Intelligent Visual Inspection for Process Improvement International. Journal of Innovative Research \& Development, Annamalai, pp. 768778 (2013)

5. Mogatti, M.: Automatic PCB Inspection algorithms: A Survey. Elsevier, Rolla, pp. 287$313(2007)$

6. Diab, S.L.: Designing a Visual Inspection System for Quality Characteristics Dimensions. In: 9th International Proceedings on Proceedings. pp. 1-2 (2010)

7. Hunter, D., Hao, Y., Pukish, M.: Selection of proper neural network sizes and architectures. IEEE Transactions on Industrial Informatics 8(2) (2012)

8. Patel, V.C.: Color Computer Vision and Artificial Neural Networks for Detection of Defects in Poultry Eggs. Artificial Intelligence Review, pp. 163-176 (1998)

9. Kesharwani, R., Dagli, C., Sun, Z.: Application of Neural Network in Shop Floor Quality Control in a Make to Order Business. Procedia Computer Science vol. 95, pp. 209216 (2016)

10. Castillo, P.A., Castellano, J.G., Merelo, J.J., Prieto, A.: Diseño de Redes Neuronales Artificiales Mediante Algoritmos Evolutivos. Revista Iberoamericana de Inteligencia Artificial no. 14, pp. 2-32 (2001) 